\title{
The spliceosomal catalytic core arose in the RNA world... or did it?
}

\author{
W Ford Doolittle
}

\section{Abstract \\ A new study adds to the evidence for a common evolutionary origin of the spliceosome and group II introns, but RNA-world questions remain.}

\section{The RNA world and its conjectured persistence}

Joseph Piccirilli and colleagues at the University of Chicago appear to have driven a final nail in the coffin of any remaining doubt that the spliceosome is, at its catalytic heart, a ribozyme. Their recent Nature article shows that U6 snRNA (a key spliceosome component) is by itself capable of catalyzing canonical splicing reactions [1]. Not unexpectedly, they conclude that the common catalytic mechanism used by the spliceosome and group II introns is consistent with a common evolutionary origin'. And they go on to claim that their findings 'support the idea that modern ribonucleoprotein enzymes evolved from a primordial "RNA world" in which catalysis was performed exclusively by RNA.' There might well have been such a world, and some modern ribozymes might still carry its heritage, but it would be wrong to think that the data of Piccirilli and colleagues lend any additional support to this already very popular evolutionary conjecture or to conclude that this conjecture is close to proven.

I will explain at the end why I offer these quibbles, but first, what did Piccirilli and colleagues show that was not known before? It has been known since the 1980s that both self-splicing group II and spliceosomal mRNA introns are excised by a two-step trans-esterification mechanism involving a branched intermediate, known as a lariat. As the former can proceed without protein, perhaps the small nuclear RNAs (snRNAs) at the core of the spliceosome were also the catalytic agent, not the hundred-plus associated splicing and auxiliary proteins. In his memorably titled one-page 1991 essay 'Five-easy pieces', Phil Sharp -

Correspondence: ford@dal.ca

Department of Biochemistry and Molecular Biology, Dalhousie University, Halifax, Nova Scotia B3H 4R2, Canada reasoning by analogy from the trans-splicing of fragmented genes in chloroplasts - speculated that the five snRNAs were fragments of an ancestral group II intron, reassembling at the beginning of each intron to form something like the ancestral catalytic ribozyme structure [2]. Spliceosomal proteins would serve merely to assist and stabilize.

Structural and functional similarities between group II and spliceosomal intron RNAs looking very like homologies continue to be documented, and, in 2001, Valadkhan and Manley showed that a protein-free complex of two snRNAs, U2 and U6, is catalytic, performing a reaction 'related to the first step of splicing' [3]. What Piccirilli's team has now done is show that U6 snRNA can catalyze the exact same two-step reaction as that of group II ribozymes, using metal ligands that 'correspond directly to catalytic metal ligands in domain $\mathrm{V}$ in structures of a group II intron'.

Although we really do not know how unlikely it is that this same structure would have been achieved twice by convergence, most biologists will probably accept this as compelling evidence that group II and spliceosomal splicing machineries are indeed related. That is, either the latter is derived from the former, the former is derived from the latter, or both descend from a common ancestor that was neither. It seems most unlikely that snRNAs - dispersed and addicted to protein helpers for assembly as they are could have gotten together into one molecule and stripped down to make a self-splicing group II intron. And an ancestral entity that was neither group II nor spliceosomal seems not to be known anywhere. So Sharp was probably right.

\section{Evolutionary forces and the derivation of introns}

Speculations over the evolutionary relationships of introns and spliceosomes to the genomes they infest have a history almost as complex as these structures themselves. Most sensible people accept Tom Cavalier-Smith's notion [4] that eukaryotes 'caught' mobile ('selfish') group II introns from the bacterial progenitor of the mitochondrion, and these then degenerated into snRNAs, recruiting multiple splicing proteins (some perhaps already engaged in stabilizing 
ribozymatic activity). All extant eukaryotic nuclear genomes, with one exception, suffer from introns, and all extant eukaryotes appear once to have had (if not still to have) mitochondria. So that all fits. And the falling apart into five pieces must have happened early, quickly and completely, if not also easily. There are no known group II introns in eukaryotic nuclear genomes and there is considerable conservation of the splicing machinery (including key spliceosomal proteins of the U2 or U12 systems) between eukaryotic lineages [5].

Work from Marlene Belfort's lab [6] identifies one force that might have persuaded recently introduced group II introns to give up their independence in eukaryotes, except in organelles, where they indeed persist. Belfort and colleagues showed that a bacterial group II intron introduced into a yeast nuclear gene renders its transcript subject to nonsense-mediated mRNA decay and (mysteriously) difficult to translate, even when spliced. Bill Martin and Eugene Koonin imagine, with much more drama, 'the existence of a turbulent phase of genome evolution in the wake of mitochondrial origin, during which group II introns invaded the host's chromosomes, spread as transposable elements into hundreds - perhaps thousands - of positions that have been conserved to the present, and fragmented into both mRNA introns and snRNA constituents of the spliceosome' [7]. For them, the host for the mitochondrial vector of the intron disease was as yet a prokaryote, and the nucleus evolved as a way to temporally separate splicing from translation, with which it would otherwise interfere.

\section{In the RNA world we trust - or should we?}

The second commonly accepted evolutionary belief about introns that Piccirilli and colleagues claim to strengthen is that their ribozymatic activity is a direct and uninterrupted inheritance from the RNA world - and indeed is evidence that such a world once existed. Maybe that world existed, but the results of Piccirilli and colleagues do not add to the evidence for it. If spliceosomal introns indeed descend from group II introns and the latter are indeed self-splicing because they inherited their catalytic core from the RNA world, then how spliceosomal introns work today is irrelevant. It's only if we believed that the catalytic activities of group II introns and spliceosomal introns descended separately from an ancestor in the RNA world that Piccirilli and coworkers would have provided further evidence for 'the idea that modern ribonucleoprotein enzymes evolved from a primordial "RNA world".

And for that matter, why do we think that the catalytic activity of group II introns is an RNA-world relic? For the ribozymal catalytic core of the ribosome, there is a very good case to be made [8]. Translation presumably preceded the appearance of modern ribosomal proteins and it strains credulity to imagine a ribozyme substituting itself at the heart of an already functioning and obviously essential proteinaceous machinery.

Not so for introns, even of group II, which the cell can take or leave. We know that RNA has extraordinary evolutionary potential, even now: venture capitalists have invested fortunes in companies developing ribozymes for therapeutic purposes. We also know that transposable elements have their own evolutionary impetus and that, in principle at least, the most successful will do the least harm. Why could not ribozymatic group II intron splicing have been invented later, in a DNA world? Curcio and Belfort [9], for instance, once mooted that perhaps 'the ability of group II introns to self-splice out of an RNA transcript developed from a reverse-splicing retroelement, owing to the selective pressure to reduce the deleterious effects of insertion' - a co-evolutionary model nicely elaborated more recently by Toor et al. [10].

\section{Relics - and making a not-so-easy peace}

That group II introns are found in both bacteria and archaea is no proof that they are truly ancient RNA retroelements: they are infectious agents capable of interdomain transfer. Certainly, that there are such ribozymatic activities is good evidence that an RNA world was possible. But it is neither compelling evidence that such a world really existed nor an indication that modern exemplars have ancient origins. Too readily do we assume that the existing entities by analogy to which we construct pre-cellular evolutionary scenarios are themselves relics of those ancient days. Relics are funny things, for all that they can resemble inferred ancestors. That they are still around suggests that there is selection to maintain them - however, such selection might also then create them de novo, even now.

\section{Competing interests}

The author declares that he has no competing interests.

\section{Acknowledgments}

I thank John Archibald and Mike Gray for comments, and the Canadian Institutes for Health Research for support.

\section{Published: 13 December 2013}

\section{References}

1. Fica SM, Tuttle N, Novak T, Nan-Sheng L, Lu J, Koodathingal P, Dai Q, Staley JP, Piccirilli JA: RNA catalyses nuclear pre-mRNA splicing. Nature 2013, 503:229-234.

2. Sharp PA: Five easy pieces. Science 1991, 254:663.

3. Valadkhan S, Manley JL: Splicing-related catalysis by protein-free snRNAs. Nature 2001, 413:701-707.

4. Cavalier-Smith T: Intron phylogeny: a new hypothesis. Trends Genet 1991, 7:145-148.

5. Rogozin IB, Carmel L, Csuros M, Koonin EV: Origin and evolution of splicesomal introns. Biol Direct 2012, 7:11.

6. Chalamcharla VR, Curcio J, Belfort M: Nuclear expression of a group II intron is consistent with splicesomal intron ancestry. Genes Dev 2010, 24:827-836.

7. Martin W, Koonin EV: Introns and the origin of nucleus-cytosol compartmentalization. Nature 2006, 440:41-42. 
8. Noller HF: Evolution of protein synthesis from an RNA world. Cold Spring Harb Perspect Biol 2012, 4:a003681.

9. Curcio MJ, Belfort M: Retrohoming: CDNA-mediated mobility of group II introns requires a catalytic RNA. Cell 1996, 84:9-12.

10. Toor N, Hausner G, Zimmerly S: Coevolution of group II intron RNA

structures with their intron-encoded reverse transcriptases. RNA 2001, 7:1142-1152.

doi:10.1186/gb4145

Cite this article as: Doolittle: The spliceosomal catalytic core arose in

the RNA world... or did it?. Genome Biology 2013 14:141. 